The writers are indebted to Dr. J. T. Irving, Head of the Physiology Department, for his interest in this work.

\title{
BIBLIOGRAPHY
}

Cantor, S. J.-Med. Jl. of Australia, Vol. I, p. 822, 1927.

FUCHS, E.-Text-book of Ophthalmology, 4th Edition, p. 305, 1911.

Gudjonsson, S.-Acta Pathol. et Microbiol. Scandinavica, Supplementum IV. 1930.

- Acta Ophthal., Vol. VIII, 1930.

Knapp, A. H.-Jl. Amer. Med. Assoc., Vol. CX, p. 1993 (June), 1938.

Parsons, J. H.-Diseases of the Eye, 7th Edition, 1934.

\section{A COMPARISON OF LENS AND SKIASCOPE METHODS IN RETINOSCOPY WITH UNDILATED PUPILS}

\author{
BY
}

W. J. B. RIDDELL

GLASGOW

For over thirty years the routine refraction work in the Ophthalmic Institution in Glasgow has been carried out by means of a circular skiascope designed by $\mathrm{H}$. Wright Thomson. The instrument consists of a sheet aluminium wheel nine inches in diameter mounted on the end of a half inch aluminium tube which is fifty inches in length. Within the circumference of the disc lenses are fitted, the number of which is conditioned by their diameter. The instrument with which most of the subsequent observations were made has thirty-six half inch lenses of the following powers : plus $8 \cdot 0,7 \cdot 0,6 \cdot 0,5 \cdot 0,4 \cdot 0,3.5,3.0,2 \cdot 75,25,2 \cdot 25,2 \cdot 0,1.75$, $1.5,1.0,0.75,0.5$ and 0.25 , followed by the corresponding minus powers in the reverse order. When in use the tube is tucked well under the arm and the disc is rotated close to the patient's eye on a plane at right angles to the visual axis. With a little practice it is not difficult to keep the lenses close to the observed eye and also to maintain the working distance at a full metre. Failure to attend to these two points leads to obvious fallacies. It is my own practice to refract the right eye with my right eye and the left one with my left. This enables the patient to fix a distant target and thereby relax his accommodation. The side of the wheel facing the observer is painted white on the plus half and black on the minus; the strength of each lens is indicated in numerals visible to him. A folding type with a telescopic tube has also been made, but the essential features of the instrument have not been changed since its introduction. In the evolutionary series of such instruments the skiascope is a simplification of 
Javal's optometer. A somewhat similar arrangement was employed in the Prentice retinoscope combined with Lionel Beale's ophthalmoscope, but the working distance was reduced to a third of a metre. The modern refracting head is in its turn derived from these instruments.

The original purpose of the device was to facilitate routine refraction work in school children. It was used by McIlroy in the investigation of the refraction of 1,335 unselected L.C.C. school children and the figures resulting from this investigation are quoted in the literature relating to refraction curves. As the method is not in general use, it seemed to me to be worth while to establish the relationship between the usual trial case method and the skiascope, before curves provided by one method could be compared with those provided by the other. If close correlation were established between the two methods, the use of the instrument would lighten the laborious work involved in the construction of refraction curves amongst unselected samples of the population.

The essential feature of the Wright Thomson skiascope is the simplicity of its construction. Anyone familiar with the refracting box and trial frame method of performing retinoscopy will find it a time-saving device of great value in doing retinoscopy with a dilated pupil. It is of particular value in restless children who are usually interested in the wheel moving round. It does not frighten them. In routine hospital refraction work under a mydriatic followed by a post-mydriatic examination the instrument has stood the test of many thousand cases in the West of Scotland. So much so that refractionists have been trained in the Ophthalmic Institution without ever having seen a refracting box. My own experience has been that the number of complaints received is no greater than in clinics where the refracting box method is employed. For teaching purposes the orthodox method is the more satisfactory because it takes longer and comparison between the light movement seen by different observers can be made without disturbing the setting of the trial lens.

The instrument has been used for a number of years in my private practice in cases where only an approximation of the refraction was required in the first instance, or prior to the use of a mydriatic. As confidence increased it was employed as an indicator to tell which lenses were required for retinoscopy by the undilated trial frame method. The readings provided by both methods were recorded and used for the subjective testing of the patient. The series of two hundred and fifty cases used in this paper were done with these objects in view. The primary purpose was not to compare one method with the other, but to act as a check in the individual case. Thus the material is as far as 
possible unbiased. An impression was formed that the skiascope readings tended to be lower than the readings made by the ordinary method. That is they appeared to be more myopic. The following calculations were made in order to find out whether or not this clinical impression was correct. The method of comparison employed was to plot the thousand readings provided by two hundred and fifty cases where the refraction was within the plusminus eight range of the instrument, against the readings found by the trial frame method. Within these limits the cases were consecutive and unselected.

It was found that in 308 readings the skiascope was one cell or more lower, i.e., more myopic, in 494 readings the same figure was recorded and in 198 the skiascope was one cell or more higher, i.e., more hypermetropic. When a large difference was found in practice, it was taken as evidence that the patient's accommodation was very active and that a mydriatic should be employed. In all cases the skiascope was used first and it is possible that greater relaxation of accommodation was obtained when the refraction was done the second time by means of trial lenses because the patients knew what was required of them. Also the fact of an instrument moving in front of the eye tends to attract the patient's attention to close range. This was the impression I got from an extensive trial of a refracting head some time ago. I have not been able to detect any gross variation in the ordinary three-cell trial frame between the front and back cell, when refracting with low powers, although the distance between the cells may be as much as one centimetre.

The instrument makes it easy to work at a full metre, a condition which cannot be fulfilled by any of the skiascope racks which have been described from time to time, unless the observer has got quite exceptionally long arms. Such racks are probably more extensively used on the Continent than in this country. By the use of this circular skiascope the labour of routine hospital refraction work can be very much reduced, and it forms a most valuable control method in undilated retinoscopies. One point which the figures cannot illustrate is the accuracy with which low degrees of astigmatism can be estimated by any one familiar with the use of the instrument. With experience it is possible to estimate the intermediate values which are not actually present. My own practice is to indicate a high or low value by the appropriate sign, after the reading has been written down. Confirmation is obtained at the post-mydriatic test in hospital, or by the usual trial frame method in private practice, if no mydriatic has been employed. Such adjustments have not been used in compiling the figures analysed.

I am indebted to Miss Francis of the Statistical Department, 
University College, London, for constructing the following table from the data provided by the two hundred and fifty patients. In each patient the Right Horizontal and Vertical Meridians and the Left Horizontal and Vertical Meridians were measured by both methods. Thus there were four sets of two hundred and fifty duplicate readings. For each set, and for the whole thousand, tables were drawn up of the lens-skiascope readings. The results are summarised in the table. In each case, with the exception of the left-horizontal, the mean of the lensskiascope differs significantly from zero, showing that the mean lens readings are definitely higher than the mean skiascope readings.

TABLE

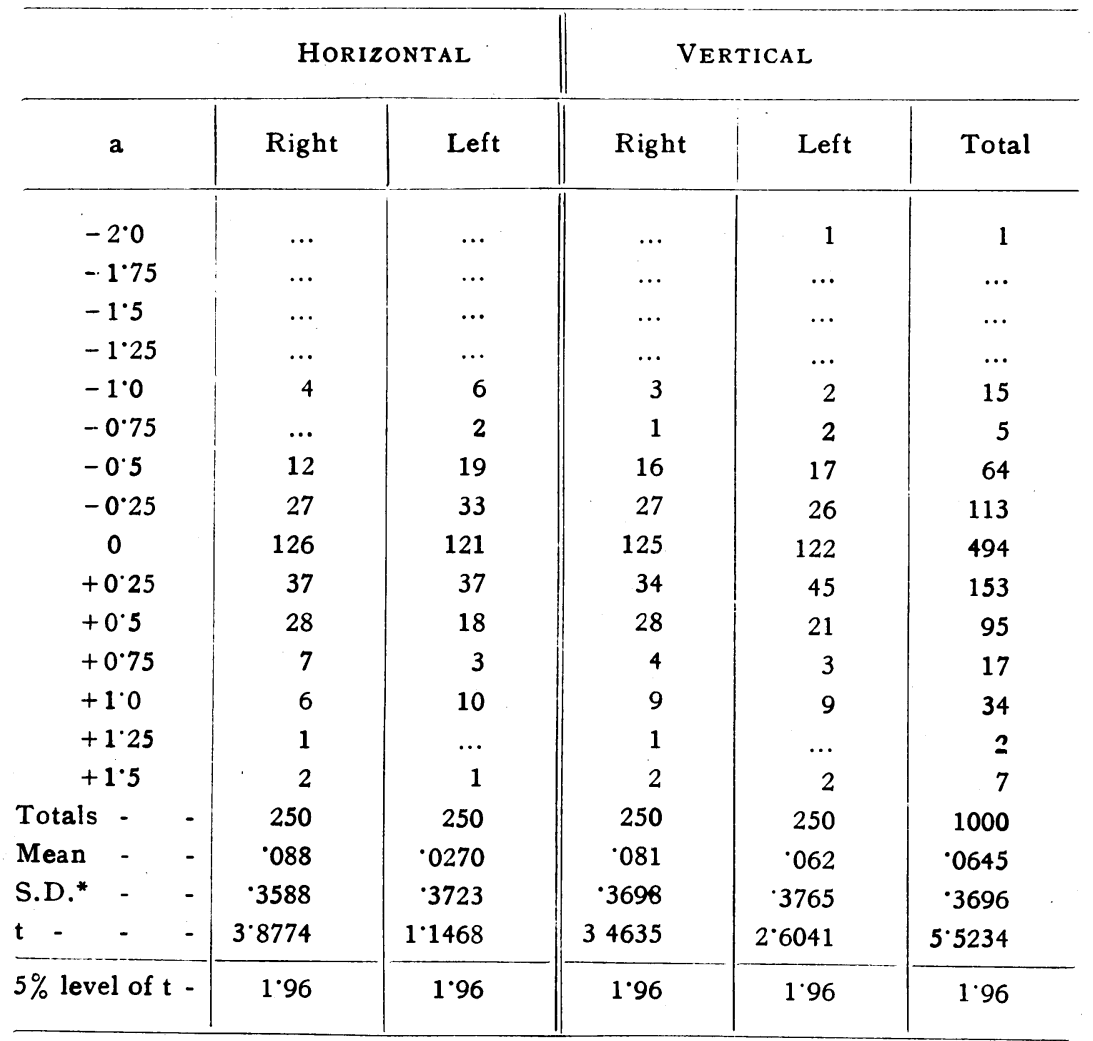

* This standard deviation is the estimated population value obtained by using the formula

the usual formula is, of course,

$$
\mathrm{O}_{x}^{-2}=\frac{\sum(x \cdot \bar{x})^{2}}{n-1}
$$

$$
\mathrm{S}^{2}=\frac{\Sigma(x-\bar{x})^{2}}{n}
$$


Repeated observations were made upon a small series of patients by the undilated lens method and it was found that in a number of readings they differed among themselves by as much as $1.0 \mathrm{D}$. 'This would indicate, of course, that the patients' accommodation was very active and would be used in practice as a warning against too great reliance upon the undilated method. Considering the total distribution of lens-skiascope readings it is seen that $10 / 1,000$ 1 per cent. lie outside -1 to +1 limits. The greatest error likely to be made by the lens method is not known, but if it is assumed to be $1.0 \mathrm{D}$, then the skiascope method appears to be comparable with the lens method since, although the individual skiascope readings tend to be lower than those of the lens method, in general only about once in a hundred times will they fall outside the limits of error which could be made by the use of lenses.

The correlation between lens and skiascope readings is very high being equal to 0.9873 . This high coefficient is to be expected since it measures the association between two series of measurements taken in the same objects. If both methods were perfect and also the observer, the correlation would be 1 . Since we have only an approximate idea as to how the lens readings may vary, we cannot draw rigid conclusions about the accuracy of the skiascope method. If the standard deviations, taken from the marginal totals, had been very much greater for the skiascope method than for the lens method, we should have suspected that this was because the skiascope method was the more variable, although the truth could not have been reached without an extensive series of repetitive measurements. However, the marginal standard deviations of the two series of readings are about the same (skiascope 2.649 and lens 2.660). While we are unable to draw any rigid inference from this, it appears that the skiascope readings are probably not much more variable than the supposedly more accurate lens readings.

Pearson curves were fitted to the distribution of lens readings and skiascope readings. They both proved to be of type IV with quite similar equations. This was to be expected as of the six curves collected by Dunstan, five were of type IV. The only exception was Wibaut's new-born distribution which was of type I and dealt with the data formed by pooling the results of four observers. As the material used for this japer was derived from patients differing widely in age and in social position no significance can be attached to the refraction curve and the detailed figures are not given.

It is with great pleasure that I acknowledge the amount of help and kindly criticism given to me by Miss David of the Statistical Department, University College, London, and the laborious calculations carried out for me by Miss Francis. 


\section{Summary}

1. A useful type of skiascope has been re-described.

2. It has been used for routine hospital refraction work for over thirty years and has proved a valuable time-saving device.

3. Figures provided by two hundred and fifty cases in which both trial frame and skiascope were used have been compared.

4. A high degree of correlation was found.

5. It is suggested that this device might be employed in the construction of refraction curves from unselected samples of the population.

6. The skiascope readings tended to be lower (i.e., more myopic) than the lens method.

\section{REFERENCES}

Dunstan, W. R.-Brit. Jl. of Ophthal., Vol. XVIII, p. 404, 1934.

McIlroy, J. Hamilton.-L.C.C. Annual Report, Vol. III, p. 86, 1928.

Thomson, H. Wright.-Ophthal. Rev., Vol. XXIV, p. 200, 1905.

\section{PATHOGENESIS OF THROMBOSIS OF THE CENTRAL RETINAL VEIN AND OF CONSECUTIVE GLAUCOMA*}

BY

Paul Weinstein, M.D.

ASSISTANT AT THE OPHTHALMOLOGICAL DEPARTMENT OF THE ALBERT APPONYI POLYCLINIC AT BUDAPEST

Glaucoma may be termed, analogously to eclampsia, a " disease of theories." Its clinical manifestation is far from being uniform, let alone its much discussed aetiology. The chief symptom is increased ocular tension, the cause whereof has been ascribed by former authors to an occlusion of the root of the iris, the circulation of the off-leading vein being impaired, and stasis developing within the eye. In opposition to this view, modern investigators look for general connections, being effective besides this local one, and believe such have been found in the system of blood-vessels. Clinical classification corresponds to the assumed connection inasmuch, as inflammatory glaucoma characterised by capillary vasoreurosis, simple glaucoma chiefly manifesting with persons suffering of arteriosclerosis, and finally glaucoma consecutive to thrombosis of the central retinal vein, called also haemorrhagic glaucoma, have been distinguished. Pathogenesis of the latter

* Research conducted by a grant of the Széchényi Scientific Associaticn. 